\title{
A functional relation for the Tornheim double zeta function
}

\author{
by \\ TAKAShi NAKAmURA (Nagoya)
}

\section{Introduction}

Definition 1.1. The Tornheim double zeta function $T(s, t, u)$, for $s, t, u$ $\in \mathbb{C}, \Re(s+u)>1, \Re(t+u)>1$ and $\Re(s+t+u)>2$, is defined by

$$
T(s, t, u):=\sum_{m, n=1}^{\infty} \frac{1}{m^{s} n^{t}(m+n)^{u}} .
$$

This function $T(s, t, u)$ is a generalization of the Riemann zeta function $\zeta(s), s \in \mathbb{C}$. Furthermore, $T(s, t, u)$ is continued meromorphically to $\mathbb{C}^{3}$ in [4]. By the definition, we have

$$
T(s, t, u)=T(t, s, u), \quad T(s, t, 0)=\zeta(s) \zeta(t) .
$$

The case of $t=0$, that is $T(s, 0, u)$, is called the Euler-Zagier double zeta function [10].

The values $T(a, b, c)$ for $a, b, c \in \mathbb{N}$ were first investigated by Tornheim [7] in 1950 and later Mordell [5] in 1958. Tornheim [7, Theorem 7] showed that $T(a, b, c)$ can be expressed as a polynomial in $\{\zeta(j) \mid 2 \leq j \leq a+b+c\}$ with rational coefficients when $a+b+c$ is odd, and that the same is true for $T(2 r, 2 r, 2 r)$ and $T(2 r-1,2 r, 2 r+1)$ [7, Theorem 8], but he did not give the coefficients. Mordell [5, Theorem III] proved that $T(2 r, 2 r, 2 r)=k_{r} \pi^{6 r}$ for some rational number $k_{r}$. In 1985 Subbarao and Sitaramachandrarao [6, Theorem 4.1] explicitly determined $T(2 p, 2 q, 2 r)+T(2 q, 2 r, 2 p)+T(2 r, 2 p, 2 q)$ $(p, q, r \in \mathbb{N})$. Then, by taking $p=q=r$, they gave an explicit formula for $T(2 r, 2 r, 2 r)(r \in \mathbb{N})$ [6, Remark 3.1]. In 1996 Huard, Williams and Zhang [3, Theorems 1-3] determined $T(r, 0, N-r)(r \in \mathbb{N}, N \in 2 \mathbb{N}+1,1 \leq r \leq N-2)$, $T(p, q, N-p-q)(p, q \in \mathbb{N} \cup\{0\}, N \in 2 \mathbb{N}+1,1 \leq p+q \leq N-1,0 \leq$ $p, q \leq N-2)$ and $T(r, r, r)(r \in \mathbb{N})$. In 2002 Tsumura [8, Theorem 1]

2000 Mathematics Subject Classification: Primary 11M41.

Key words and phrases: Tornheim double zeta function, Euler-Zagier double zeta function. 
proved that $T(p, q, r)+(-1)^{p} T(p, r, q)+(-1)^{p+r} T(r, q, p)$ is a polynomial in $\{\zeta(k) \mid 2 \leq k \leq p+q+r\}$ with rational coefficients for $p, q, r \in \mathbb{N} \cup\{0\}$ with $p+q \geq 2$ and $r \geq 2$. Recently, Espinosa and Moll provided an explicit formula for $T(x, y, z), x, y, z \in \mathbb{R}$, in terms of integrals involving Hurwitz zeta functions (see [2, Proposition 2.1 and Theorem 2.4]). Also in 2006 Tsumura [9, Theorem 4.5] proved the following functional relation:

$$
\begin{aligned}
= & 2 \sum_{\substack{j=0 \\
j \equiv a(2)}}^{a}\left(2^{1-a+j}-1\right) \zeta(a-j) \sum_{l=0}^{j / 2} \frac{(i \pi)^{2 l}}{(2 l) !}\left(\begin{array}{c}
b-1+j-2 l \\
j-2 l
\end{array}\right) \zeta(b+j+s-2 l) \\
& -4 \sum_{\substack{j=0 \\
j \equiv a(2)}}^{a}\left(2^{1-a+j}-1\right) \zeta(a-j) \sum_{l=0}^{(j-1) / 2} \frac{(i \pi)^{2 l}}{(2 l+1) !} \sum_{\substack{k=0 \\
k \equiv b(2)}}^{b} \zeta(b-k) \\
& \times\left(\begin{array}{c}
k-1+j-2 l \\
j-2 l-1
\end{array}\right) \zeta(k+j+s-2 l)
\end{aligned}
$$

(where (2) means mod 2), for $a, b \in \mathbb{N} \cup\{0\}, b \geq 2, s \in \mathbb{C}$, except for the singular points of each side of this formula.

In this paper, we prove the following result.

TheOREm 1.2. For all $a, b \in \mathbb{N}$ and $s \in \mathbb{C}$ except for the singular points, we have

$$
\begin{aligned}
T(a, b, s) & +(-1)^{b} T(b, s, a)+(-1)^{a} T(s, a, b) \\
= & \frac{2}{a ! b !} \sum_{k=0}^{\max (a, b) / 2}\left\{a\left(\begin{array}{c}
b \\
2 k
\end{array}\right)+b\left(\begin{array}{c}
a \\
2 k
\end{array}\right)\right\}(a+b-2 k-1) !(2 k) ! \\
& \times \zeta(2 k) \zeta(a+b+s-2 k) .
\end{aligned}
$$

This functional relation is considerably simpler than that of Tsumura. We are not aware of a direct proof which shows that the right-hand sides of (1.2) and (1.3) are the same. "Mathematica 5.0" shows that they are equal for all $1 \leq a \leq b \leq 100, a, b \in \mathbb{N}$. It therefore seems unlikely that a non-trivial functional relation can be deduced by equating (1.2) and (1.3).

In Section 3, we obtain new proofs of formulas for the special values of $T(a, b, c), a, b, c \in \mathbb{N}$ mentioned in the introduction by using the functional relation (1.3).

2. Proof of Theorem 1.2. Firstly, we define $\log t, t \in \mathbb{C}$, and $t^{s}$, $s, t \in \mathbb{C}$, by

$$
\log t:=\log |t|+i \arg t, \quad t^{s}:=e^{s \log t}, \quad 0 \leq \arg t<2 \pi .
$$


And for $s, t, u \in \mathbb{C}, \Re(s+u)>1, \Re(t+u)>1$ and $\Re(s+t+u)>2$, we put

$$
S(s, t, u):=\sum_{\substack{m \neq 0, n \neq 0 \\ m+n \neq 0}} \frac{1}{m^{s} n^{t}(m+n)^{u}} .
$$

Lemma 2.1. For all $a, b \in \mathbb{N}$ and $s \in \mathbb{C}$ except for the singular points, we have

$$
\begin{aligned}
S(a, b, s)= & \left(1+e^{-\pi i(a+b+s)}\right) \\
& \times\left(T(a, b, s)+(-1)^{b} T(b, s, a)+(-1)^{a} T(s, a, b)\right) .
\end{aligned}
$$

Proof. Let

$$
\begin{aligned}
& T_{1}(a, b, s):=\sum_{m, n>0} \frac{1}{m^{a} n^{b}(m+n)^{s}}=T(a, b, s), \\
& T_{2}(a, b, s):=\sum_{\substack{m<0, n>0 \\
n>-m}} \frac{1}{m^{a} n^{b}(m+n)^{s}}=\sum_{\substack{m, n>0 \\
n>m}} \frac{1}{(-m)^{a} n^{b}(n-m)^{s}} \\
& =(-1)^{-a} \sum_{m, k>0} \frac{1}{m^{a}(m+k)^{b} k^{s}}=(-1)^{-a} T(s, a, b), \\
& T_{3}(a, b, s):=\sum_{\substack{m<0, n>0 \\
-m>n}} \frac{1}{m^{a} n^{b}(m+n)^{s}}=\sum_{\substack{m, n>0 \\
m>n}} \frac{1}{(-m)^{a} n^{b}(n-m)^{s}} \\
& =e^{-\pi i(a+s)} \sum_{n, k>0} \frac{1}{(n+k)^{a} n^{b} k^{s}}=e^{-\pi i(a+s)} T(b, s, a), \\
& T_{4}(a, b, s):=\sum_{m, n<0} \frac{1}{m^{a} n^{b}(m+n)^{s}}=e^{-\pi i(a+b+s)} T(a, b, s), \\
& T_{5}(a, b, s):=\sum_{\substack{m>0, n<0 \\
-n>m}} \frac{1}{m^{a} n^{b}(m+n)^{s}}=\sum_{\substack{m, n>0 \\
n>m}} \frac{1}{m^{a}(-n)^{b}(m-n)^{s}} \\
& =e^{-\pi i(b+s)} T(s, a, b), \\
& T_{6}(a, b, s):=\sum_{\substack{m>0, n<0 \\
m>-n}} \frac{1}{m^{a} n^{b}(m+n)^{s}}=\sum_{\substack{m, n>0 \\
m>n}} \frac{1}{m^{a}(-n)^{b}(m-n)^{s}} \\
& =(-1)^{-b} T(b, s, a) \text {. }
\end{aligned}
$$

Obviously we have

$$
\sum_{j=1}^{6} T_{j}(a, b, s)=S(a, b, s) .
$$

This implies (2.2). We can also see that the convergence of $S(a, b, s)$ is equivalent to the convergence of $T(a, b, s)$. 
Lemma 2.2 ([11]). For $\Re(s)>1, \Re(t)>1$ and $\Re(u)>1$, we have

$$
S(s, t, u)=\int_{0}^{1} \sum_{m \neq 0} \frac{e^{2 \pi i m x}}{m^{s}} \sum_{n \neq 0} \frac{e^{2 \pi i n x}}{n^{t}} \sum_{l \neq 0} \frac{e^{-2 \pi i l x}}{l^{u}} d x .
$$

Proof. By putting $l=m+n$, we have

$$
\begin{aligned}
S(s, t, u) & =\sum_{\substack{m, n, l \neq 0 \\
m+n=l}} \frac{1}{m^{s} n^{t} l^{u}}=\sum_{m, n, l \neq 0} \int_{0}^{1} \frac{e^{2 \pi i(m+n-l) x}}{m^{s} n^{t} l^{u}} d x \\
& =\int_{0}^{1} \sum_{m, n, l \neq 0} \frac{e^{2 \pi i(m+n-l) x}}{m^{s} n^{t} l^{u}} d x=\int_{0}^{1} \sum_{m \neq 0} \frac{e^{2 \pi i m x}}{m^{s}} \sum_{n \neq 0} \frac{e^{2 \pi i n x}}{n^{t}} \sum_{l \neq 0} \frac{e^{-2 \pi i l x}}{l^{u}} d x .
\end{aligned}
$$

Changing the order of summation and integration is justified by absolute convergence.

We denote by $B_{j}(x)$ the Bernoulli polynomial of order $j$ defined by

$$
\frac{t e^{x t}}{e^{t}-1}=\sum_{j=0}^{\infty} B_{j}(x) \frac{t^{j}}{j !}, \quad|t|<2 \pi .
$$

It is known (see $[1$, p. 266, (22), and p. 267, (24)]) that

$$
\begin{aligned}
B_{2 j}:=B_{2 j}(0) & =(-1)^{j+1} 2(2 j) !(2 \pi)^{-2 j} \zeta(2 j), \quad j \in \mathbb{N}, \\
B_{j}(x) & =-\frac{j !}{(2 \pi i)^{j}} \lim _{K \rightarrow \infty} \sum_{\substack{k=-K \\
k \neq 0}}^{K} \frac{e^{2 \pi i k x}}{k^{j}}, \quad j \in \mathbb{N} .
\end{aligned}
$$

For $k \in \mathbb{Z}, j \in \mathbb{N}$ we have

$$
\int_{0}^{1} e^{-2 \pi i k x} B_{j}(x) d x= \begin{cases}0, & k=0, \\ -(2 \pi i k)^{-j} j !, & k \neq 0 .\end{cases}
$$

In fact, the case of $k=0$ is obvious, and in the case of $k \neq 0$, we get (2.6) by using (2.5). Next we quote [1, p. 276, 19(b)], for $p+q \geq 2$, which is

$$
\begin{aligned}
& B_{p}(x) B_{q}(x) \\
= & \sum_{k=0}^{\max (p, q) / 2}\left\{p\left(\begin{array}{c}
q \\
2 k
\end{array}\right)+q\left(\begin{array}{c}
p \\
2 k
\end{array}\right)\right\} \frac{B_{2 k} B_{p+q-2 k}(x)}{p+q-2 k}-(-1)^{p} \frac{p ! q !}{(p+q) !} B_{p+q} .
\end{aligned}
$$


Proof of Theorem 1.2. Firstly, we assume $a, b \geq 2,1+e^{-\pi i(a+b+s)} \neq 0$ and $\Re(s)>1$. By using (2.6) and (2.7), we have

$$
\begin{aligned}
-\int_{0}^{1} B_{a}(x) B_{b}(x) \sum_{l \neq 0} \frac{e^{-2 \pi i l x}}{l^{s}} d x \\
=-\int_{0}^{1} \sum_{k=0}^{\max (a, b) / 2}\left\{a\left(\begin{array}{c}
b \\
2 k
\end{array}\right)+b\left(\begin{array}{c}
a \\
2 k
\end{array}\right)\right\} \frac{B_{2 k} B_{a+b-2 k}(x)}{a+b-2 k} \sum_{l \neq 0} \frac{e^{-2 \pi i l x}}{l^{s}} d x \\
=\sum_{k=0}^{\max (a, b) / 2}\left\{a\left(\begin{array}{c}
b \\
2 k
\end{array}\right)+b\left(\begin{array}{c}
a \\
2 k
\end{array}\right)\right\} \frac{(a+b-2 k-1) ! B_{2 k}}{(2 \pi i)^{a+b-2 k}} \\
\quad \times \zeta(a+b+s-2 k)\left(1+e^{-\pi i(a+b+s)}\right) .
\end{aligned}
$$

Because of (2.2), (2.4) and (2.5), we obtain (1.3) in this region. By analytic continuation, we have (1.3) for all $a, b \in \mathbb{N}, a, b \geq 2$ and $s \in \mathbb{C}$ except for the singular points of each side of this formula.

Next we consider the case of $a=1, b \geq 2$. For $a, b \in \mathbb{N}, a, b \geq 2$ and $s \in \mathbb{C}$ except for the singular points, we define $K(a, b, s)$ by the right-hand side of (1.3). We quote some basic properties $[3,(1.5)]$ proved by easy computations, for $s, t, u \in \mathbb{C}$ except for the singular points:

$$
\left\{\begin{array}{l}
T(s, t-1, u+1)+T(s-1, t, u+1)=T(s, t, u), \\
T(s, t+1, u-1)-T(s-1, t+1, u)=T(s, t, u), \\
T(s+1, t, u-1)-T(s+1, t-1, u)=T(s, t, u) .
\end{array}\right.
$$

For $b \geq 2$, we have

$$
\begin{aligned}
K(2, b, s)= & T(2, b, s)+(-1)^{b} T(b, s, 2)+(-1)^{2} T(s, 2, b) \\
= & T(1, b, s+1)+(-1)^{b} T(b, s+1,1)+(-1) T(s+1,1, b) \\
& +T(2, b-1, s+1)+(-1)^{b-1} T(b-1, s+1,2) \\
& +(-1)^{2} T(s+1,2, b-1)
\end{aligned}
$$

by (2.8) and the result in the case $a, b \geq 2$ which we have already shown. Hence we have to show

$$
K(2, b, s)=K(1, b, s+1)+K(2, b-1, s+1), \quad b \geq 2 .
$$

In fact we have

$$
\begin{aligned}
\frac{2}{b !}\left\{\left(\begin{array}{c}
b \\
2 k
\end{array}\right)+b\left(\begin{array}{c}
1 \\
2 k
\end{array}\right)\right\}+\frac{2}{2 !(b-1) !}\left\{2\left(\begin{array}{c}
b-1 \\
2 k
\end{array}\right)+(b-1)\left(\begin{array}{c}
2 \\
2 k
\end{array}\right)\right\} \\
=\frac{2}{2 ! b !}\left\{2\left(\begin{array}{c}
b \\
2 k
\end{array}\right)+b\left(\begin{array}{c}
2 \\
2 k
\end{array}\right)\right\}(b+1-2 k), \quad 0 \leq k \leq b / 2 .
\end{aligned}
$$

In the cases of $k=0,1, b / 2$, we have this equation immediately. For $2 \leq k \leq$ 
$(b-1) / 2$, we obtain it by

$$
b\left(\begin{array}{c}
b-1 \\
l
\end{array}\right)=\frac{b(b-1) \cdots(b-l+1)(b-l)}{l !}=(b-l)\left(\begin{array}{l}
b \\
l
\end{array}\right), \quad 0 \leq l \leq b .
$$

We can prove (1.3) for the case of $a=b=1$ similarly.

3. New proofs of known formulas. In this section, from our theorem we deduce formulas for the special values of $T(a, b, c)(a, b, c \in \mathbb{N})$ mentioned in the introduction. By taking $a=2 p, b=2 q, s=2 r$ in (1.3), we have $T(2 p, 2 q, 2 r)+T(2 q, 2 r, 2 p)+T(2 r, 2 p, 2 q)$

$$
\begin{aligned}
= & \frac{2}{(2 p) !(2 q) !} \sum_{k=0}^{\max (p, q)}\left\{2 p\left(\begin{array}{c}
2 q \\
2 k
\end{array}\right)+2 q\left(\begin{array}{c}
2 p \\
2 k
\end{array}\right)\right\}(2 p+2 q-2 k-1) !(2 k) ! \\
& \times \zeta(2 k) \zeta(2 p+2 q+2 r-2 k) .
\end{aligned}
$$

This formula coincides with [6, Theorem 4.1]. (There is a misprint in $[6$, Theorem 4.1], "min" is to be replaced by "max".) Putting $a=b=s=r$ in (1.3) we have, after easy computations of binomial coefficients,

$$
T(r, r, r)=\frac{4}{1+2(-1)^{r}} \sum_{k=0}^{r / 2}\left(\begin{array}{c}
2 r-2 k-1 \\
2 k-1
\end{array}\right) \zeta(2 k) \zeta(3 r-2 k) .
$$

This formula is [3, Theorem 3].

For $a, b, c \in \mathbb{N}$, we define $N(a, b, c)$ as half of the right-hand side of (1.3). We recall the harmonic product formula

$$
T(a, 0, b)+T(b, 0, a)=\zeta(a) \zeta(b)-\zeta(a+b) .
$$

Putting $s=0$ in (1.3) and multiplying by $(-1)^{a}$, we obtain

$$
(-1)^{a} \zeta(a) \zeta(b)+(-1)^{a+b} T(b, 0, a)+T(a, 0, b)=2(-1)^{a} N(a, b, 0) .
$$

When $a+b \in 2 \mathbb{N}+1$, we can remove $T(b, 0, a)$ by summing the above two formulas. Hence

$$
T(a, 0, b)=-\frac{\zeta(a+b)}{2}+\frac{1+(-1)^{b}}{2} \zeta(a) \zeta(b)+(-1)^{a} N(a, b, 0)
$$

for all $a, b \geq 2, a+b \in 2 \mathbb{N}+1$. Next by changing the variables in (1.3), we obtain

$$
\left\{\begin{array}{l}
(-1)^{b} T(a, b, c)+T(b, c, a)+(-1)^{c} T(c, a, b)=2 N(b, c, a), \\
(-1)^{a} T(a, b, c)+(-1)^{c} T(b, c, a)+T(c, a, b)=2 N(c, a, b) .
\end{array}\right.
$$

In the case of $a+b+c \in 2 \mathbb{N}+1$, we can remove $T(b, c, a)$ and $T(c, a, b)$ by multiplying the former equality by $(-1)^{b}$ and the latter by $(-1)^{a}$, and summing the resulting formulas. Hence we have

$$
T(a, b, c)=(-1)^{b} N(b, c, a)+(-1)^{a} N(c, a, b), \quad a+b+c \in 2 \mathbb{N}+1 .
$$


By putting $s=t=1$ in the first equation of (2.8), we obtain

$$
T(1,1, u)=2 T(1,0, u+1) .
$$

Hence we can calculate $T(1,0, c+1)$ if $c+1 \in 2 \mathbb{N}$. Therefore we obtain another proof of $[3$, Theorems 1, 2]. Moreover we get

$$
T(p, q, r)+(-1)^{p} T(p, r, q)+(-1)^{p+r} T(r, q, p)=2(-1)^{p} N(p, r, q)
$$

by taking $a=p, b=r$ and $s=q$ in (1.3), and multiplying by $(-1)^{p}$. Hence we obtain another proof of $[8$, Theorem 1$]$, because $N(p, q, r)$ is a polynomial in $\{\zeta(k) \mid 2 \leq k \leq p+q+r\}$ with rational coefficients for $p, q, r \in \mathbb{N} \cup\{0\}$ with $p+q \geq 2$ and $r \geq 2$.

Acknowledgments. I thank Professors Kohji Matsumoto and Hirofumi Tsumura for useful advice.

\section{References}

[1] T. M. Apostol, Introduction to Analytic Number Theory, Springer, 1976.

[2] O. Espinosa and V. H. Moll, The evaluation of Tornheim double sums. I, J. Number Theory 116 (2006), 200-229.

[3] J. G. Huard, K. S. Williams, and Z. Y. Zhang, On Tornheim's double series, Acta Arith. 75 (1996), 105-117.

[4] K. Matsumoto, On the analytic continuation of various multiple zeta-functions, in: Number Theory for the Millennium, II (Urbana, IL, 2000), M. A. Bennett et al. (eds.), A K Peters, 2002, 417-440.

[5] L. J. Mordell, On the evaluation of some multiple series, J. London Math. Soc. 33 (1958), 368-371.

[6] M. V. Subbarao and R. Sitaramachandrarao, On some infinite series of L. J. Mordell and their analogues, Pacific J. Math. 119 (1985), 245-255.

[7] L. Tornheim, Harmonic double series, Amer. J. Math. 72 (1950), 303-314.

[8] H. Tsumura, On some combinatorial relations for Tornheim's double series, Acta Arith. 105 (2002), 239-252.

[9] -, On functional relations between the Mordell-Tornheim double zeta functions and the Riemann zeta function, Math. Proc. Cambridge Philos. Soc., to appear; Tokyo Metropolitan Univ. Preprint Ser., 2006, No. 2, 11 pp.

[10] D. Zagier, Values of zeta functions and their applications, in: First European Congress of Mathematics, Vol. II (Paris, 1992), Progr. Math. 120, Birkhäuser, 1994, $497-512$.

[11] —, Introduction to multiple zeta values, lectures at Kyushu Univ., 1999.

Graduate School of Mathematics

Nagoya University

Chikusa-ku, Nagoya, 464-8602, Japan

E-mail: m03024z@math.nagoya-u.ac.jp

Received on 2.2.2006

and in revised form on 30.7.2006 